Vol. 3, No. 2, 2017

\author{
Jerzy Jaroszewicz ${ }^{1}$, Olesya Maksymovych ${ }^{2}$, Andrii Dzyubyk ${ }^{3}$, Ihor Nazar ${ }^{4}$ \\ ${ }^{1}$ Department of Production Management, Faculty of Management, Bialystok University of Technology, \\ 2, O. Tarasiuka Str., Kleosin, 16-001, Poland, E-mail: j.jaroszewicz@ pb.edu.pl \\ ${ }^{2}$ Department of Welding Manufacture, Diagnostics and Restoration of Metal Structures, Lviv Polytechnic \\ National University, 12, S. Bandery Str., Lviv, Ukraine, E-mail: olesyamax@meta.ua \\ ${ }^{3}$ Department of Welding Manufacture, Diagnostics and Restoration of Metal Structures, Lviv Polytechnic \\ National University, 12, S. Bandery Str., Lviv, Ukraine, E-mail: dar.lviv@gmail.com \\ ${ }^{4}$ Department of Welding Manufacture, Diagnostics and Restoration of Metal Structures, Lviv Polytechnic \\ National University, 12, S. Bandery Str., Ukraine, Lviv, E-mail: nazari@ukr.net
}

\title{
DETERMINATION OF STRESSES AND ULTIMATE LOADS FOR COMPOSITE PLATES WITH ELASTIC INCLUSIONS
}

Received: October 28, 2017 / Revised: December 20, 2017 / Accepted: December 26, 2017

(C) Maksymovych O., Dzyubyk A., Nazar I., Jaroszewicz J., 2017

Abstract. In the article, the algorithm for determination of stresses in anisotropic plates with elastic inclusions of another anisotropic material was developed on the basis of complex singular integral equations. The solving of integral equations has been carried out numerically using the method of mechanical quadratures. The strength analysis (calculation of strength) of composite plates with inclusions has been performed using the Hoffman criterion.

Keywords: stress concentration factors; anisotropic plate; elastic inclusion; stress-strain state; singular integral equation; strength analysis; Hoffman criterion.

\section{Introduction and Review of Modern Information Sources}

The method of boundary integral equations is widely used to study the stress-strain state of isotropic and anisotropic plates with holes. If the loads on the holes boundaries are prescribed, such problems have been sufficiently studied already [1]-[4]. The problems of determination of the stress-strain state of anisotropic plates with inclusions have been studied to a lesser extent. For studying isotropic plates with inclusions, the singular integral equations are being used. For these equations, the numerical methods allow to seek a solution with controllable accuracy [5].

In publications [4], [6]-[7], the interdependencies between the potentials of Lehnitskiy and the stresses and displacements have been determined. Based on these interdependencies and on the Cauchy theorem, the modified singular integral equations for anisotropic plates with holes are written in the simplified form. In the paper, the analogous integral equations for plates with sealed-in elastic inclusions are obtained. The efficiency and simplicity of use of the developed numerical algorithm are exemplified by calculation of stresses at inclusions of various shapes and by determination of the ultimate loads.

\section{Problem Statement}

In order to assess the strength of composite materials with inclusions, it is necessary to investigate the stresses in the neighbourhood of the section boundary, where, as a rule, the fracture nucleates. Since in this case all the components of the stress tensor are non-zero, the calculation of strength of the inclusion regions adjacent to the section boundary and the matrix should be performed on the basis of general criteria for a planar stressed state. In addition, it is necessary to separately assess the strength of the material, which is directly adjacent to the boundary, which, as a rule, has the strength properties different from the matrix ones.

\section{Main Material Presentation}

Let us consider the Hoffman strength criterion for composite materials, which is necessary for further calculations and takes into account the difference between the ultimate stresses of tension and 
compression. Let us consider the specimen of the rectangular shape made of the chosen composite material and being under the influence of bidirectional tension caused by forces $\sigma_{1}, \sigma_{2}$ and of shear caused by forces $\tau_{12}$. According to the Hoffman strength criterion, the fracture takes place if the stresses satisfy the following condition [8]:

$$
-\frac{\sigma_{1}^{2}}{X_{c} X_{t}}+\frac{\sigma_{1} \sigma_{2}}{X_{c} X_{t}}-\frac{\sigma_{2}^{2}}{Y_{c} Y_{t}}+\frac{X_{c}+X_{t}}{X_{c} X_{t}} \sigma_{1}+\frac{Y_{c}+Y_{t}}{Y_{c} Y_{t}} \sigma_{2}+\frac{\tau_{12}^{2}}{S_{12}^{2}}=1
$$

where $X_{t}, Y_{t}$ are the ultimate values of stresses when tensioning the specimen along the orthotropy axes being parallel to the coordinate axes $O x$ and $O y ; X_{c}, Y_{c}$ are the analogous values of stresses when compressing the specimen; $S_{12}$ are the ultimate values of tangential stresses for pure (simple) shear.

These constants and the mechanical characteristics, which are determined on the basis of corresponding experiments [8], for fiberglass are presented in Table 1.

Mechanical and Strength Characteristics of the fiberglass

Table 1

\begin{tabular}{|c|c|}
\hline Characteristic & Value \\
\hline$E_{1}, \mathrm{GPa}$ & 54 \\
\hline$E_{2}, \mathrm{GPa}$ & 18 \\
\hline$v_{12}$ & 0.25 \\
\hline$G_{12}, \mathrm{GPa}$ & 9 \\
\hline$X_{t}, \mathrm{MPa}$ & 1035 \\
\hline$Y_{t}, \mathrm{MPa}$ & 28 \\
\hline $\mathrm{S}, \mathrm{MPa}$ & 41 \\
\hline$X_{c}, \mathrm{MPa}$ & 1035 \\
\hline$Y_{c}, \mathrm{MPa}$ & 138 \\
\hline
\end{tabular}

Let us adopt that the stress in the plate is defined by one load parameter $p$ (for example, by the value of forces tensioning the plate). Further, there will be used the algorithm, in which the stresses on the section boundary may be presented as follows: $\sigma_{n}=p S_{n}, \sigma_{t}=p S_{t}, \tau_{n t}=p S_{n t}$, where $\sigma_{n}, \sigma_{\theta}, \tau_{n \theta}$ are the normal, hoop (circumferential) and tangential stresses near the section boundary; $S_{n}, S_{\theta}, S_{n \theta}$ are the known values non-dependable on parameter $p$. Let us determine the stresses in Cartesian coordinate system, which axes are parallel to the axes of orthotropy. For this, let us use the relations written in matrix form:

where

$$
\left(\begin{array}{c}
\sigma_{x} \\
\sigma_{y} \\
\tau_{x y}
\end{array}\right)=\Lambda\left(\begin{array}{l}
\sigma_{n} \\
\sigma_{t} \\
\tau_{n t}
\end{array}\right),
$$

$$
\Lambda=\left(\begin{array}{ccc}
\cos ^{2} \alpha & \sin ^{2} \alpha & -\sin (2 \alpha) \\
\sin ^{2} \alpha & \cos ^{2} \alpha & \sin (2 \alpha) \\
\frac{\sin (2 \alpha)}{2} & -\frac{\sin (2 \alpha)}{2} & \cos (2 \alpha)
\end{array}\right)
$$

$\alpha$ is the angle between the normal to the section boundary and the axis $O x$.

Based on the presented equations, we obtain $\sigma_{x}=p F_{1}, \quad \sigma_{y}=p F_{2}, \quad \tau_{x y}=p F_{12}$, where $\left(F_{1}, F_{2}, F_{12}\right)^{\prime}=\Lambda\left(S_{n}, S_{t}, S_{n t}\right)^{\prime}$. 
Thus, on the basis of equation (1), we obtain the following equation for determination of the parameter $p$ :

where

$$
f_{1} p^{2}+f_{2} p-1=0
$$

$$
f_{1}=-\frac{F_{1}^{2}}{X_{c} X_{t}}+\frac{F_{1} F_{2}}{X_{c} X_{t}}-\frac{F_{2}^{2}}{Y_{c} Y_{t}}+\frac{F_{12}^{2}}{S_{12}^{2}} ; \quad f_{2}=\frac{X_{c}+X_{t}}{X_{c} X_{t}} F_{1}+\frac{Y_{c}+Y_{t}}{Y_{c} Y_{t}} F_{2} .
$$

Let us adopt the smaller positive value of the equation root as the ultimate load $P$ for the point. Let us denote the smallest value of $p$ on the section boundary of the inclusions by $p_{1}$ and of the matrix by $p_{2}$. The strength of the material adjacent to the inclusion boundary, as a rule, is determined on the basis of stresses normal to the section boundary taking into account the condition $\sigma_{n}<p_{n}$, where $p_{n}$ is the known value defined on the basis of experimental testing and depended on the technology of composites production. Let us define the minimal value of the quantity $1 / S_{n}$ on the section boundary and denote it by $p_{3}$. In this case, the ultimate value of load parameter may be determined by the formula: $p_{\partial o n}=\min \left(p_{1}, p_{2}, p_{3}\right)$.

Thus, the presented relations allow carrying out the calculations of strength when the distribution of the stressed state of piecewise homogeneous plates is known.

The algorithm of determination of the stress-strain state of anisotropic plates with elastic inclusions. The problem of determination of the stress-strain state of an anisotropic plate with elastic inclusion of another material is considered. Let us adopt that the plate is under the influence of the loading, which is applied at infinity; the plate is in the conditions of a flat stressed state. A similar problem for isotropic plates is well studied in literature. For anisotropic materials, such problems were considered in [9] using the series method. In this paper, a numerical algorithm for solving this problem is developed. It is based on the method of integral equations and allows to consider a system of inclusions of almost arbitrary form. Let us assume that there is a perfect mechanical contact on the boundary of materials sections.

General relations. Let us consider an arbitrary curve $\Gamma$ lying in the region D occupied by the plate. The complex potentials of Lehnitskiy, the vectors of stresses $(X, Y)$ and the derivatives of displacements $(u, v)$ on this curve are related by the dependence [4]:

$$
\begin{gathered}
\Phi\left(z_{1}\right)=\frac{-V+s_{1} \cdot U+p_{1} \cdot X+q_{1} \cdot Y}{\Delta_{1} \cdot z_{1}^{\prime}} ; \\
\Psi\left(z_{2}\right)=\frac{-V+s_{2} \cdot U+p_{2} \cdot X+q_{2} \cdot Y}{\Delta_{2} \cdot z_{2}^{\prime}},
\end{gathered}
$$

where $\quad U=d u / d s ; \quad V=d v / d s ; \quad z_{j}=x+s_{j} y ; \quad u^{\prime}=d u / d s ; \quad v^{\prime}=d v / d s ; \quad z_{j}^{\prime}=d x / d s+s_{j} d y / d s ;$ $p_{j}=a_{11} s_{j}^{2}+\alpha_{12}-\alpha_{16} s_{j} ; q_{j}=\alpha_{12} s_{j}+\alpha_{22} / s_{j}-\alpha_{26} ; j=1,2 ; d s$ is the differential of the arc on $\Gamma$; $\Delta_{1}=\alpha_{11}\left(s_{1}-s_{2}\right)\left(s_{1}-\overline{s_{1}}\right)\left(s_{1}-\overline{s_{2}}\right) ; \Delta_{2}=\alpha_{11}\left(s_{2}-s_{1}\right)\left(s_{2}-\overline{s_{1}}\right)\left(s_{2}-\bar{s}_{2}\right) ; \alpha_{i j}$ are the elastic constants; $s_{1,2}$ are the roots of the equation $\alpha_{11} s^{4}-2 \alpha_{16} s^{3}+\left(2 \alpha_{12}+\alpha_{66}\right) s^{2}-2 \alpha_{26} s+\alpha_{22}=0$.

In order to construct the general solution, let us consider separately the internal and infinite regions limited by the closed contour $\Gamma$. Using the properties of analytical functions for potentials in infinite region, let us write the following representation (transform) [4]:

$$
\begin{gathered}
\Phi\left(z_{1}\right)=\frac{1}{2 \pi i} \int_{\Gamma_{1}} \frac{\Phi_{\Gamma}\left(t_{1}\right) d t_{1}}{t_{1}-z_{1}}+\Phi_{S}\left(z_{1}\right) ; \\
\Psi\left(z_{2}\right)=\frac{1}{2 \pi i} \int_{\Gamma_{2}} \frac{\Psi_{\Gamma}\left(t_{2}\right) d t_{2}}{t_{2}-z_{2}}+\Psi_{S}\left(z_{2}\right),
\end{gathered}
$$

where $\Phi_{\Gamma}$ and $\Psi_{\Gamma}$ are the ultimate values of potentials; $\Gamma^{(1)}$ and $\Gamma^{(2)}$ are the contours in auxiliary coordinate systems $\left(x_{1}, y_{1}\right)$ and $\left(x_{2}, y_{2}\right)$, into which the curve $\Gamma$ transits after affine transformation 
(mapping) [9]; $\Phi_{S}\left(z_{1}\right)$ and $\Psi_{S}\left(z_{2}\right)$ are the complex potentials for infinite plate, which are corresponding to the load applied in infinity (the positive direction of integration is chosen as clockwise one). Using the considered representation and the representation (2), we obtain:

$$
\begin{gathered}
\Phi\left(z_{1}\right)=\frac{1}{2 \pi \Delta_{1} i} \int_{\Gamma} \frac{\left(-V+s_{1} U+p_{1} X+q_{1} Y\right) d s}{t_{1}-z_{1}}+\Phi_{S}\left(z_{1}\right) ; \\
\Psi\left(z_{2}\right)=\frac{1}{2 \pi \Delta_{2} i} \int_{\Gamma} \frac{\left(-V+s_{2} U+p_{2} X+q_{2} Y\right) d s}{t_{2}-z_{2}}+\Psi_{S}\left(z_{2}\right) .
\end{gathered}
$$

Let us substitute the potentials (3) into the formulas for determination of the vector of stresses and the derivatives of displacements on the contour $\Gamma$ [4]. Using the Sokhotski formulas, after performing transformations on the contour $\Gamma$, we obtain:

$$
\begin{gathered}
2 \operatorname{Re}\left[\Phi\left(z_{1}\right) z_{1}^{\prime}+\Psi\left(z_{2}\right) z_{2}^{\prime}\right]=-Y / 2 ; \quad 2 \operatorname{Re}\left[s_{1} \Phi\left(z_{1}\right) z_{1}^{\prime}+s_{2} \Phi\left(z_{2}\right) z_{2}^{\prime}\right]=X / 2 ; \\
2 \operatorname{Re}\left[p_{1} \Phi\left(z_{1}\right) z_{1}^{\prime}+p_{2} \Psi\left(z_{2}\right) z_{2}^{\prime}\right]=U / 2, \quad 2 \operatorname{Re}\left[q_{1} \Phi\left(z_{1}\right) z_{1}^{\prime}+q_{2} \Psi\left(z_{2}\right) z_{2}^{\prime}\right]=V / 2 .
\end{gathered}
$$

where $\Phi\left(z_{1}\right), \Psi\left(z_{2}\right)$ are the complex potentials (3), in which the Couchy integrals are considered in the sense of principal value.

The analogous relations take place for internal region. The only difference is the necessity to change the direction of integration, i.e., to consider the anticlockwise direction.

Discrete interrelations between the stresses and the displacements on the contour. Using the quadrature formulas $[4 ; 10]$ for integrals included in representation (3) at $z_{j}=z_{j v}$, we obtain:

$$
\begin{aligned}
& \Phi\left(z_{\mathrm{lv}}\right)=H \sum_{k=1}^{N} s_{k}^{\prime} \frac{A_{1} U_{k}+A_{2} V_{k}+A_{3} X_{k}+A_{4} Y_{k}}{t_{1 k}-z_{\mathrm{l}}}+\Phi_{S}\left(z_{\mathrm{lv}}\right) ; \\
& \left.\Psi_{\left(z_{2 v}\right.}\right)=H \sum_{k=1}^{N} s_{k}^{\prime} \frac{B_{1} U_{k}+B_{2} V_{k}+B_{3} X_{k}+B_{4} Y_{k}}{t_{2 k}-z_{2 v}}+\Psi_{S}\left(z_{2 v}\right),
\end{aligned}
$$

where $X_{k}=X\left(T_{k}\right) ; Y_{k}=Y\left(T_{k}\right) ; U_{k}=U\left(T_{k}\right) ; V_{k}=V\left(T_{k}\right) ; z_{j}=x_{v}+s_{j} y_{v} ; t_{j k}=x_{k}+s_{j} y_{k} ; j=1,2$; $A_{1}=-\frac{i p_{1}}{2 \pi \Delta_{1}} ; \quad A_{2}=-\frac{i q_{1}}{2 \pi \Delta_{1}} ; \quad B_{1}=-\frac{i p_{2}}{2 \pi \Delta_{2}} ; \quad B_{2}=-\frac{i q_{2}}{2 \pi \Delta_{2}} ; T_{k}$ and $Z_{v}$ are the point with coordinates

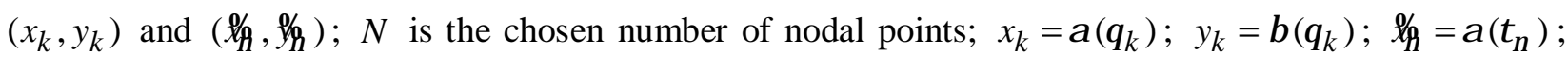
$\Re \rho=\beta\left(\tau_{v}\right) ; \theta_{k}=H k ; H=2 \pi / N ; \tau_{v}=\theta_{v}-H / 2 ; s_{k}^{\prime}=s^{\prime}\left(\theta_{k}\right) ; s^{\prime}(\theta)=\sqrt{\alpha^{\prime}(\theta)^{2}+\beta^{\prime}(\theta)^{2}}$.

Using the formulas (5) and taking into account (4), we obtain the interrelations between the vector of stresses and the derivatives of displacements on the contour:

$$
\begin{gathered}
U_{v} / 2=H \sum_{k=1}^{N} s_{k}^{\prime}\left(U_{k} U_{v k}^{(1)}+V_{k} U_{v k}^{(2)}+X_{k} U_{v k}^{(3)}+Y_{k} U_{v k}^{(4)}\right)+U_{S v} ; \\
V_{v} / 2=H \sum_{k=1}^{N} s_{k}^{\prime}\left(U_{k} V_{v k}^{(1)}+V_{k} V_{v k}^{(2)}+X_{k} V_{v k}^{(3)}+Y_{k} V_{v k}^{(4)}\right)+V_{S v} ; \\
X_{v} / 2=H \sum_{k=1}^{N} s_{k}^{\prime}\left(U_{k} X_{v k}^{(1)}+V_{k} X_{v k}^{(2)}+X_{k} X_{v k}^{(3)}+Y_{k} X_{v k}^{(4)}\right)+X_{S v} ; \\
Y_{v} / 2=H \sum_{k=1}^{N} s_{k}^{\prime}\left(U_{k} Y_{v k}^{(1)}+V_{k} Y_{v k}^{(2)}+X_{k} Y_{v k}^{(3)}+Y_{k} Y_{v k}^{(4)}\right)+Y_{S v},
\end{gathered}
$$


where $v=1, \ldots, N ;\left(U_{v k}^{(j)}, V_{v k}^{(j)}\right)$ and $\left(X_{v k}^{(j)}, Y_{v k}^{(j)}\right)$ are the derivatives of the displacements vector

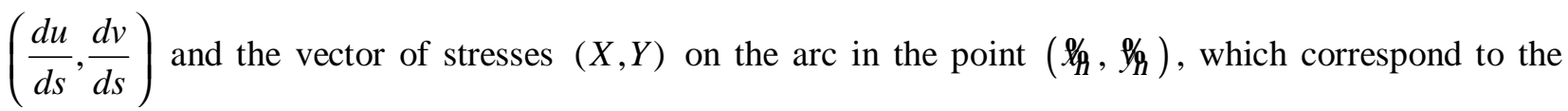
potentials:

$$
\Phi\left(z_{1}\right)=\frac{A_{j}}{t_{1 k}-z_{1}} ; \quad \Psi\left(z_{2}\right)=\frac{B_{j}}{t_{2 k}-z_{2}} .
$$

The coefficients $\left(U_{S V}, V_{S V}\right)$ and $\left(X_{S V}, Y_{S V}\right)$ are the derivatives of the displacements vector and the vector of stresses on the arc $\Gamma$ in the point $(\wp, \Re)$, which correspond to the potentials $\Phi_{S}, \Psi_{S}$.

The relations (4) and (6) are valid for internal region, if we substitute the multiplier $-H$ instead of $H$ everywhere before the sums and instead of $\Phi_{S}\left(z_{1}\right)$ and $\Psi_{S}\left(z_{2}\right)$ we adopt the complex potentials for infinite plate, which correspond to the concentrated forces applied to the internal region.

Piecewise-homogeneous plate. Let us consider an infinite plate containing an elastic inclusion. Let us denote by $L$ the contour lying on the boundary of materials section. Let us denote the constants and solutions for inclusion and matrix with a help of indexes 1 and 2, correspondingly. Let us take into account the fact that on the section boundary the derivatives of the displacements vector and the vector of stresses are equal. Let us denote them as $U, V, X, Y$. Let us write down the relations (6) for the inclusion and the matrix (as the contour $\Gamma$, let us take the curve $L$ ) and equalize the expressions, which are in the right sides of theses formulas. As a result, the following system of equations will be obtained:

$$
\begin{aligned}
& \sum_{k=1}^{N} s_{k}^{\prime}\left(U_{k} \alpha_{v k}^{(1)}+V_{k} \alpha_{v k}^{(2)}+X_{k} \alpha_{v k}^{(3)}+Y_{k} \alpha_{v k}^{(4)}\right)=a_{v} \\
& \sum_{k=1}^{N} s_{k}^{\prime}\left(U_{k} \beta_{v k}^{(1)}+V_{k} \beta_{v k}^{(2)}+X_{k} \beta_{v k}^{(3)}+Y_{k} \beta_{v k}^{(4)}\right)=b_{v} \\
& \sum_{k=1}^{N} s_{k}^{\prime}\left(U_{k} \gamma_{v k}^{(1)}+V_{k} \gamma_{v k}^{(2)}+X_{k} \gamma_{v k}^{(3)}+Y_{k} \gamma_{v k}^{(4)}\right)=c_{v} \\
& \sum_{k=1}^{N} s_{k}^{\prime}\left(U_{k} \delta_{v k}^{(1)}+V_{k} \delta_{v k}^{(2)}+X_{k} \delta_{v k}^{(3)}+Y_{k} \delta_{v k}^{(4)}\right)=d_{v}
\end{aligned}
$$

where $\quad v=1, \ldots, N ; \quad \alpha_{v k}^{(j)}=U_{v k}^{j, 1}+U_{v k}^{j, 2} ; \quad \beta_{v k}^{(j)}=V_{v k}^{j, 1}+V_{v k}^{j, 2} ; \quad \gamma_{v k}^{(j)}=X_{v k}^{j, 1}+X_{v k}^{j, 2} ; \quad \delta_{v k}^{(j)}=Y_{v k}^{j, 1}+Y_{v k}^{j, 2} ;$ $a_{v}=\frac{U_{S V}^{(1)}-U_{S V}^{(2)}}{H} ; b_{v}=\frac{V_{S V}^{(1)}-V_{S V}^{(2)}}{H} ; c_{v}=\frac{X_{S V}^{(1)}-X_{S V}^{(2)}}{H} ; d_{v}=\frac{Y_{S V}^{(1)}-Y_{S V}^{(2)}}{H}$.

The coefficients $U_{v k}^{j, m}, V_{v k}^{j, m}, X_{v k}^{j, m}, Y_{v k}^{j, m}$ for $m=1$ may be defined as coefficients $U_{v k}^{(j)}, V_{v k}^{(j)}$, $X_{v k}^{(j)}, Y_{v k}^{(j)}$ presented in (6) for elastic constants with inclusions, and for $m=2$ - for matrix.

The calculation of stresses and assessment of strength of the plates with elastic inclusions. At first, let us the case of elliptical inclusion, when the plate is under the influence of constant load applied on the infinity $\sigma_{x}^{\infty}, \sigma_{y}^{\infty}, \tau_{x y}^{\infty}$; the semiaxis of ellipse lie on the coordinate axes and are equal to $r_{1}, r_{2}$. The exact solution of this problem has been obtained in closed form in [9].

The comparison of the stresses calculated with a help of developed algorithm and according to the analytical solutions, indicates its high accuracy and efficiency.

In Fig. 1, there are presented the results of calculation of the relative stresses on the section boundary in the isotropic plate for the inclusion with sides ratio of $1 / 2$ when $v_{1}=v_{2}=0,3$ and the elasticity modulus ratio of $E_{1}=2 E_{2}$ (the inclusion is more rigid). In Fig. 1, there are presented the results for the same conditions when $E_{1}=0,5 E_{2}$. 


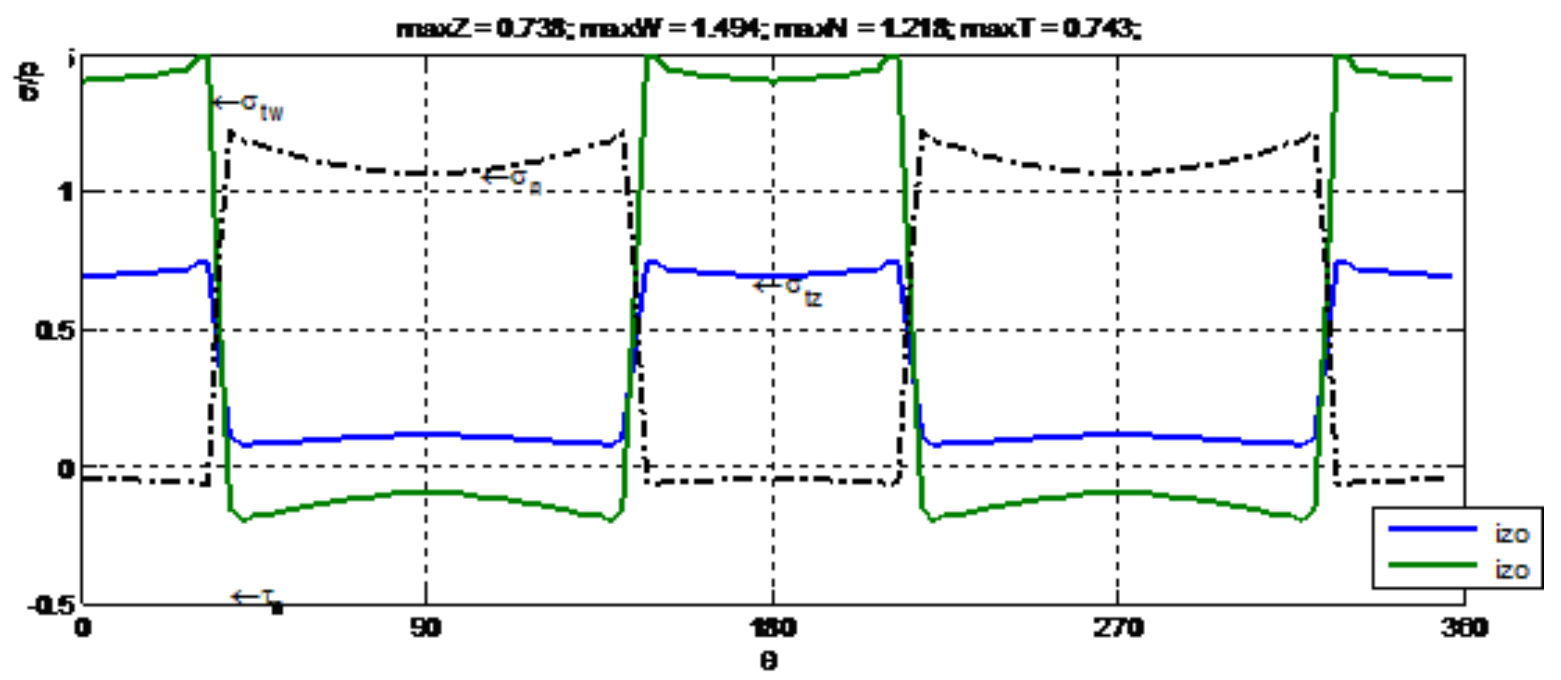

Fig. 1. The stresses in the plane with rectangular inclusions for $b=0,5 a$ during the tensioning of the isotropic plate when $E_{1}=2 E_{2}$

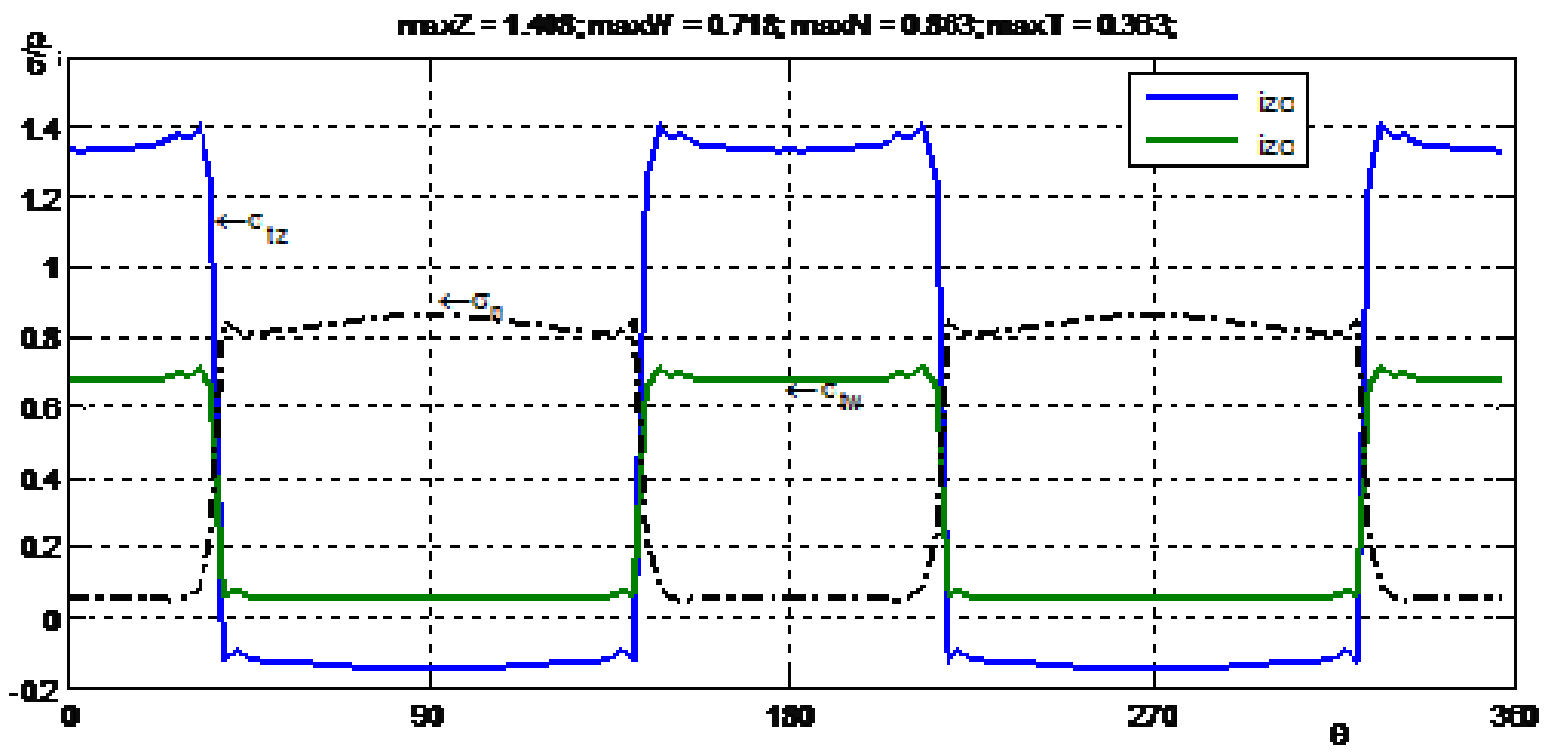

Fig. 2. The stresses in the plane with rectangular inclusions for $b=0,5 a$ during the tensioning of the isotropic plate when $E_{1}=0,5 E_{2}$

On the basis of the obtained results (Figs. 1-2), we may conclude that hoop (circumferential) stresses on the section boundary have the largest values in the matrix when the inclusion is softer, whereas the stresses are the largest in the inclusion when it is more rigid.

Let us carry out the calculation of strength of the glass-epoxide plate with rectangular isotropic inclusion with half-sides $a$ and $b$ for the ratio of $b / a=0,5$ and with rounded corners of quarter-circle shape of the radius of $0,5 b$ during the single-direction tension by the loads $p$ along the $O y$ axis when the rigidity (stiffness) of the material is maximal. Fig. 3 presents the results of the stresses calculation on the section boundary (continuous lines) and the value $p_{1}$ (dashed line) for the constant elastic inclusions when $v=0,3, E=25 \mathrm{GPa}$ (Fig. 3, a) and $E=50 \mathrm{GPa}$ (Fig. 3, b). The analogous results for $E=75 \mathrm{GPa}$ (Fig. 3, $a$ ) and $E=100 \mathrm{GPa}$ are presented in Fig. 4.

The ultimate loads for the cases considered above are as follows: $0,17 Y_{t}, 0,8 Y_{t}, 0,46 Y_{t}, 0,28 Y_{t}$, where $Y_{t}$ are the ultimate loads for homogeneous glass-epoxide plate, which are equal $1035 \mathrm{MPa}$. It is necessary to mention that the fracture nucleates near the rounded corners. On the basis of the obtained 
results, one may see that the maximal ultimate values of loads are in the case when $E=50 \mathrm{GPa}$. It is necessary to mention that such value is similar to the elasticity modulus $E_{y}$ of the considered material. The analogous results have been defined for the circular inclusion. The obtained ultimate values of loads are equal $0,2 Y_{t}, 0,78 Y_{t}, 0,54 Y_{t}, 0,35 Y_{t}$, i.e., are similar to the values for the rectangular inclusions calculated above.

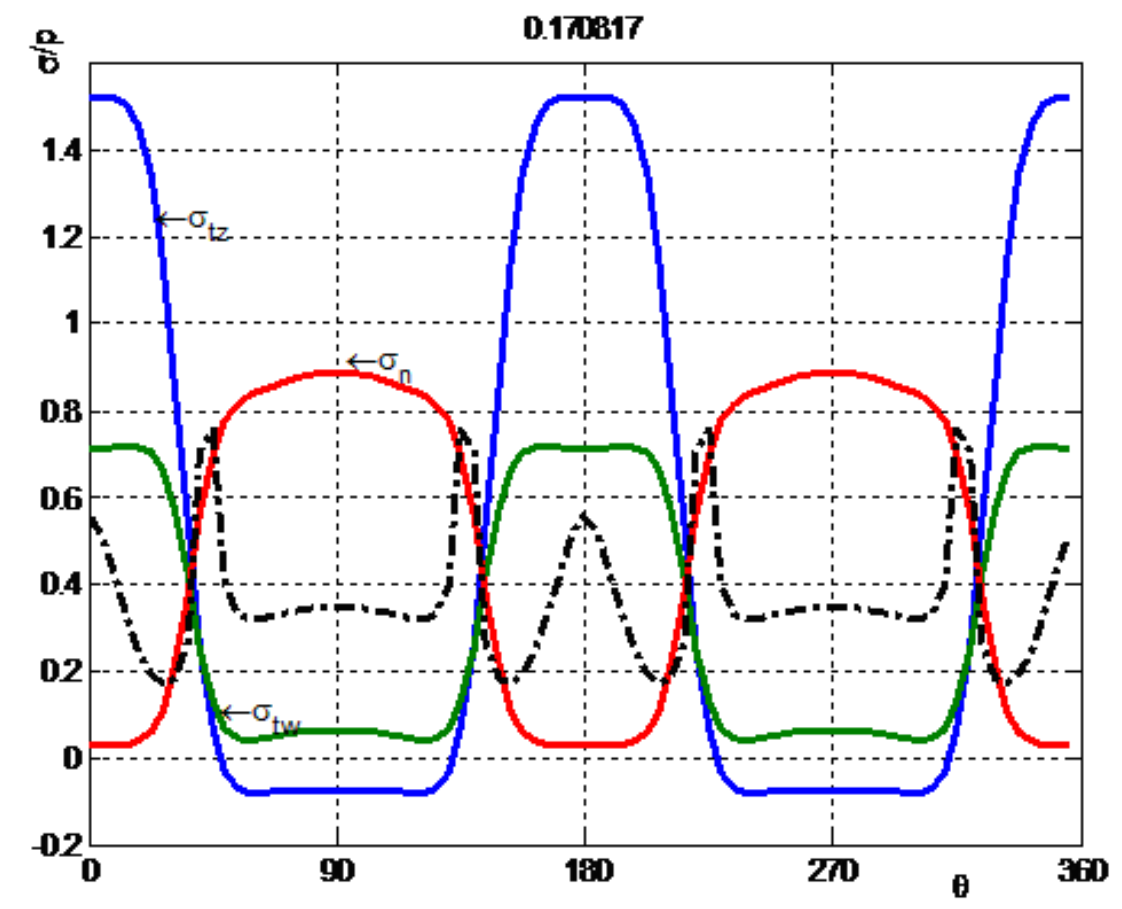

$a$

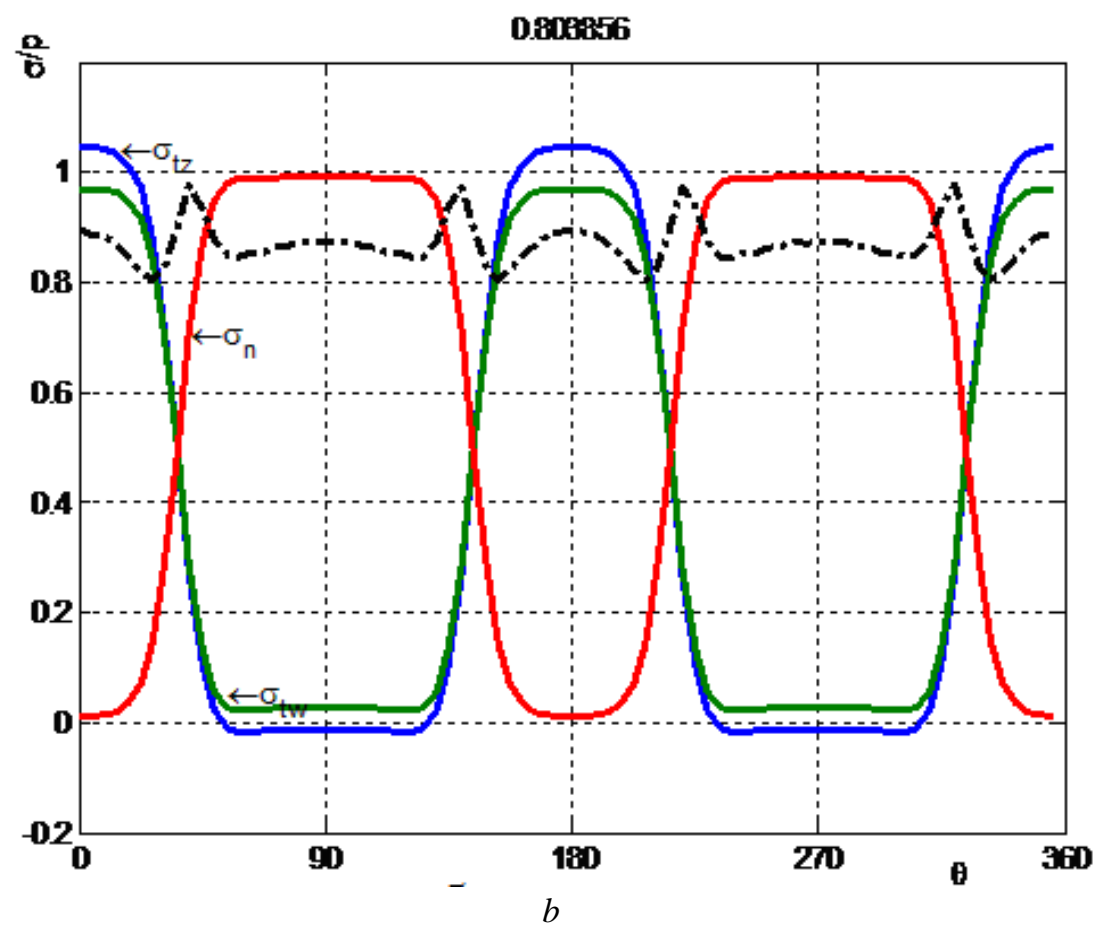

Fig. 3. The stresses in the plane with rectangular isotropic inclusion when $E=25 \mathrm{GPa}(a)$ and $E=50 \mathrm{GPa}$ (b) 


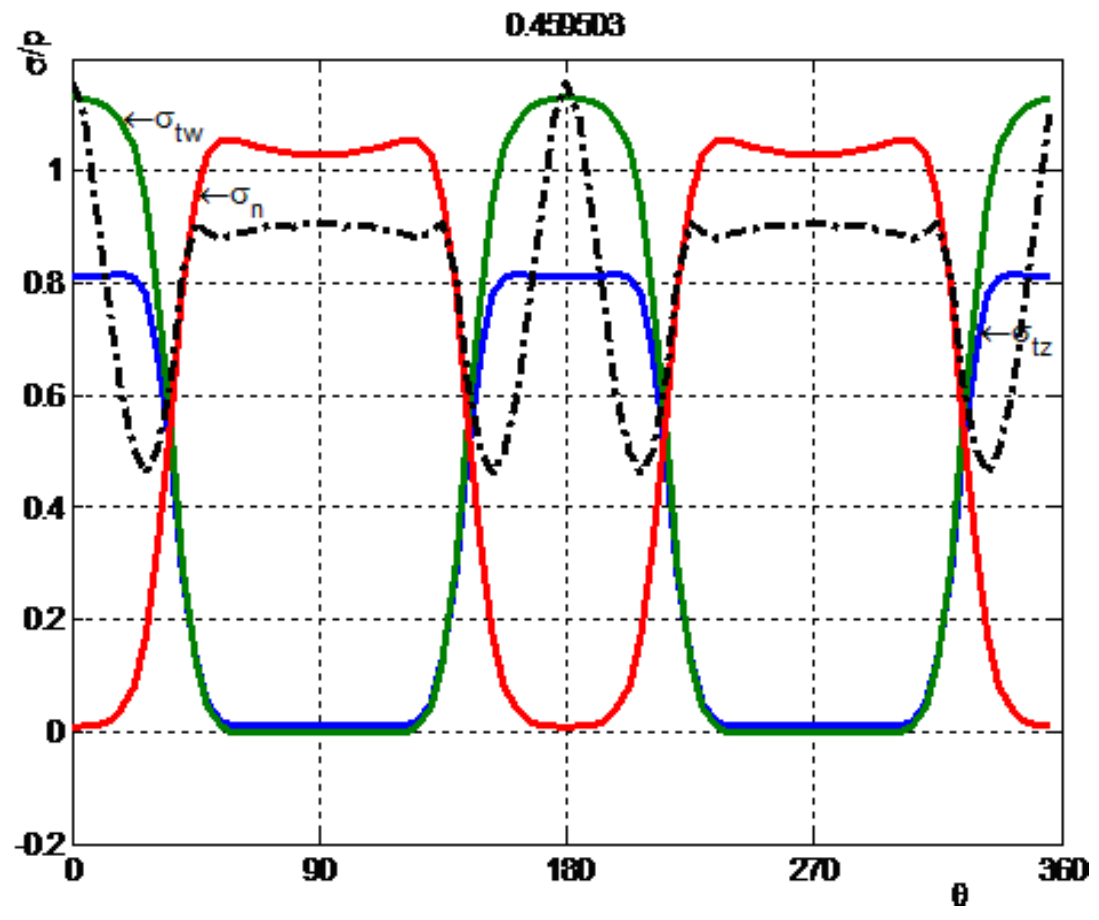

$a$

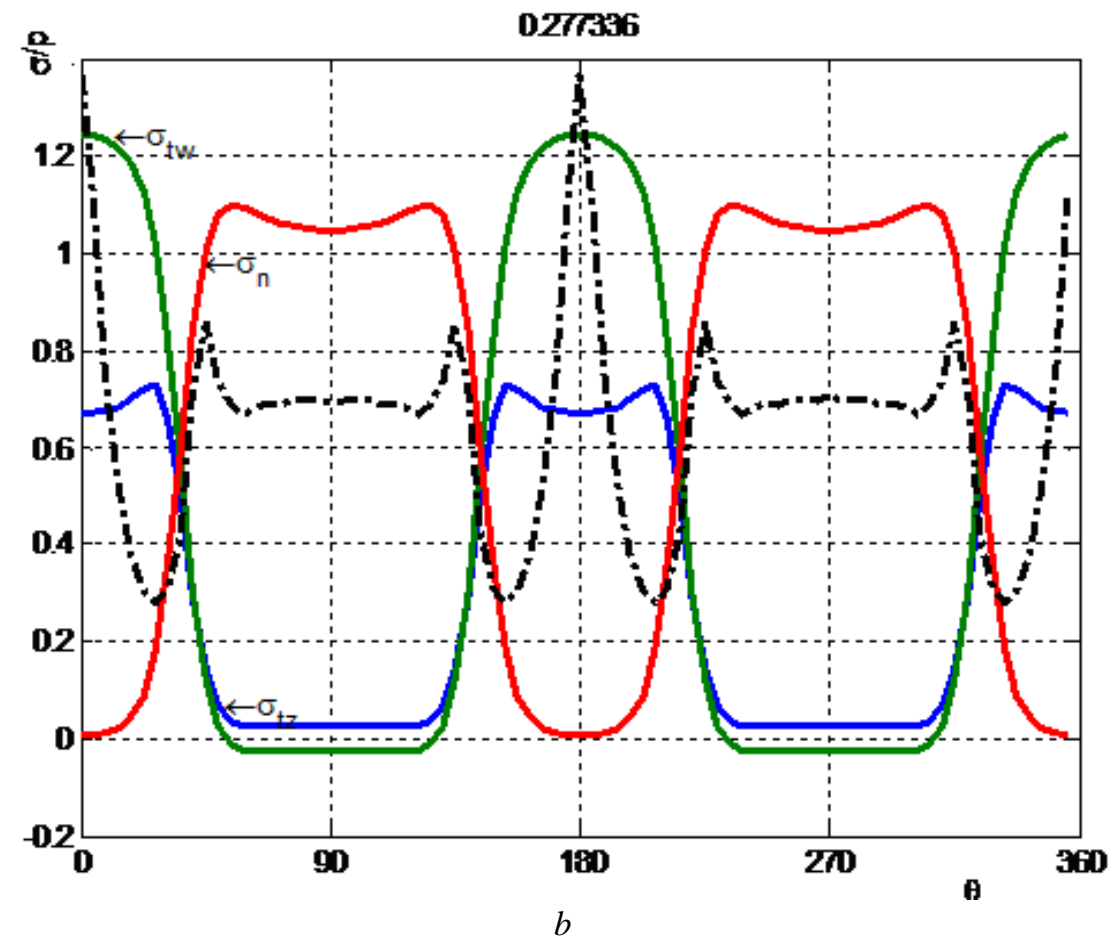

Fig. 4. The stresses in the plane with rectangular isotropic inclusion when $E=75 \mathrm{GPa}(a)$ and $E=100 \mathrm{GPa}(b)$

\section{Conclusions}

The singular integral equations of the elasticity theory for anisotropic plates with elastic inclusions are written in complex form. The equations were derived on the basis of established interrelations between the potentials of Lekhnitski and the stresses and displacements. The integral equations solving was carried out using the method of mechanical quadratures and was reduced to solving of the system of linear algebraic equations. On the basis of the developed algorithm, the investigation of stresses near the 
inclusions in composite plates with different levels of anisotropy was carried out. The calculation of ultimate loads for composite plates with inclusions was performed.

\section{References}

[1] C. A Brebbia and J. Dominguez, "Boundary Elements an Introductory Course", Me Graw-Hill: Computational Mechanics Publ., 1989.

[2] C. A Brebbia and J. Dominguez, "Boundary Elements Analysis", Berlin: Springer-Verlag Publ., 2007.

[3] N. A. Schclar, "Anisotropic Analysis Using Boundary Elements", Southampton: Computational Mechanics Publ., 1994.

[4] O. Maksymovych, "Stress calculation and optimization in composite plates with holes based on the modified integral equation method", Engineering Analysis with Boundary Elements, vol. 83, pp. 180-187, 2017.

[5] M. P Savruk and A. Kazberuk, "A unified approach to problems of stress concentration near V-shaped notches with sharp and rounded tip", Int. J. Appl. Mech., vol. 43, no. 2, pp. 182-197, 2007.

[6] O. Maksymovych, et al., "Doubly periodic cracks in the anisotropic medium with the account of contact of their faces", Acta mechanica et automatica, vol. 8, no. 3., pp. 160-164, 2014.

[7] V. V. Bozhydarnyk and O. V. Maksymovych, "Pruzhna rivnovaha anizotropnoi pivploshchyny z periodychnoiu systemoiu otvoriv i trishchyn" ["Elastic equilibrium of anisotropic half-plane with periodic system of holes and cracks"], Fizyko-Khimichna Mekhanika Materialiv [Materials Science], no.6, pp. 15-21, 2001. [in Ukrainian].

[8] R. M. Jones, "Mechanics of Composite Materials". Philadelphia, PA: Taylor and Francis Publ., 1999.

[9] S. G. Lekhnitskii, "Anisotropic Plates". New York: Gordon and Breach Science Publ., 1987.

[10] M. M. Chawla and T. R. Ramakrishnan, "Numerical evaluation of integrals of periodic functions with Cauchy and Poisson type kernels", "Numerische Mathematik", vol. 22, issue 4, pp. 317-323, 1974. 